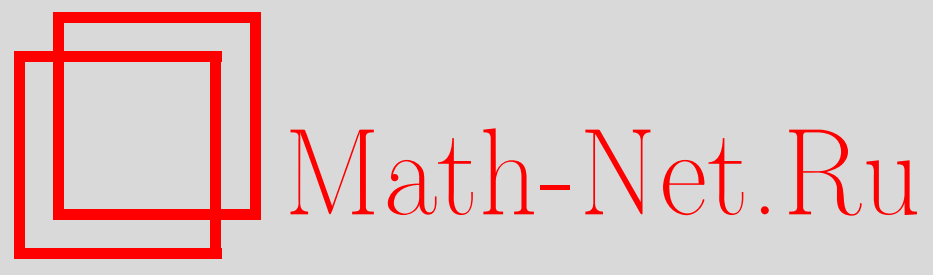

А. В. Нагаев, Асимптотические свойства многомерных устойчивых плотностей и несимметричные проблемы больших уклонений, Теория вероятн. и ее примен., 2006, том 51, выпуск 4, 691-711

DOI: https://doi.org/10.4213/tvp20

Использование Общероссийского математического портала Math-Net.Ru подразумевает, что вы прочитали и согласны с пользовательским соглашением

http://www.mathnet.ru/rus/agreement

Параметры загрузки:

IP : 44.207 .124 .84

26 апреля 2023 г., 17:09:56

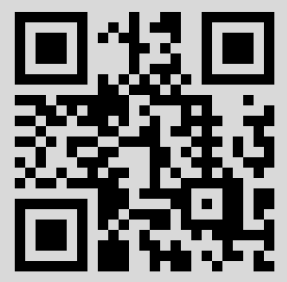




\title{
АСИМПТОТИЧЕСКИЕ СВОЙСТВА МНОГОМЕРНЫХ УСТОЙЧИВЫХ ПЛОТНОСТЕЙ И НЕСИММЕТРИЧНЫЕ ПРОБЛЕМЫ БОЛЬШИХ УКЛОНЕНИЙ
}

\begin{abstract}
Рассматриваются асимптотические свойства так называемых односторонних многомерных устойчивых распределений, обладающих тем свойством, что минимальный выпуклый конус, порожденный носителем пуассоновской спектральной меры, не совпадает c $\mathbf{R}^{d}$. Плотность такого распределения по некоторым направлениям может убывать чрезвычайно быстро. С помощью техники сопряженных распределений Крамера устанавливается точная асимптотика и выписывается асимптотический ряд, описывающий характер этого убывания.
\end{abstract}

Ключевые слова и фразы: $\alpha$-устойчивое распределение, строго $\alpha$-устойчивое распределение, односторонний устойчивый закон, преобразование Крамера, преобразование Лежандра-Фенхеля, пуассоновская спектральная мера, сопряженное распределение.

\section{1. Введение}

Класс так называемых $\alpha$-устойчивых распределений играет очень важную роль в теории вероятностей и ее приложениях. В значительной мере потому, что они возникают в качестве предельных для сумм независимых одинаково распределенных случайных векторов. Существуют различные определения $\alpha$-стабильности. Для наших целей удобно определять $\alpha$-стабильность следующим образом. Рассмотрим независимые одинаково распределенные случайные векторы $\eta, \eta^{(1)}, \eta^{(2)}, \ldots$. Будем говорить, что распределение $\eta$ является $\alpha$-устойчивылм в $\mathbf{R}^{d}$, если для любого $n>1$ существуют действительное число $b_{n}>0$ и $d$-мерный неслучайный вектор $\mathbf{a}^{(n)}$ такие, что

$$
\eta \stackrel{d}{=} b_{n}\left(\eta^{(1)}+\cdots+\eta^{(n)}\right)+\mathbf{a}^{(n)}
$$

Если в $(1.1) \mathbf{a}^{(n)} \equiv \mathbf{0}$ для всех $n>1$, то $\alpha$-устойчивое распределение называется строго $\alpha$-устойчивылм.

\footnotetext{
* Университет Николая Коперника, Торунь, Польша.
} 
Рассмотрим случайный вектор $\eta$, имеющий $\alpha$-устойчивое распределение, не являющееся гауссовским. Обозначим через $s(x)$ плотность этого распределения. Его характеристическая функция $\widehat{s}(t)$ допускает следующее представление (см., например, замечание 2.3 .3 в [1]):

$$
\ln \widehat{s}(t)=\imath\langle\gamma, t\rangle+\alpha \int_{S^{d-1}} \int_{0}^{\infty}\left(\mathrm{e}^{\imath r\langle e, t\rangle}-1-\imath \frac{r\langle e, t\rangle}{1+r^{2}}\right) r^{-\alpha-1} \mu(\mathrm{d} e) \mathrm{d} r,
$$

где параметр $\alpha$, называемый характеристическим показателем, лежит в промежутке $(0,2)$, а $\mu$ - конечная мера на борелевских подмножествах единичной сферы $S^{d-1}$. Всюду далее мы называем $\mu$ пуассоновской спектральной мерой или просто спектральной мерой. Символом $\langle\cdot, \cdot\rangle$ обозначается скалярное произведение в $\mathbf{R}^{d}$.

Представление (1.2) может быть преобразовано в следующие зачастую более удобные представления:

$$
-\ln \widehat{s}(t)=\left\{\begin{array}{c}
\imath\left\langle\gamma^{\prime}, t\right\rangle+\alpha \int_{S^{d-1}}|\langle t, e\rangle|^{\alpha}\left(1-\imath \operatorname{tg}\left(\frac{\alpha \pi}{2}\right) \operatorname{sign}\langle t, e\rangle\right) \mu(\mathrm{d} e), \\
\text { если } \alpha \neq 1, \\
\imath\left\langle\gamma^{\prime}, t\right\rangle+\alpha \int_{S^{d-1}}|\langle t, e\rangle|\left(1+\imath \frac{2}{\pi} \operatorname{sign}\langle t, e\rangle \ln |\langle t, e\rangle|\right) \mu(\mathrm{d} e), \\
\text { если } \alpha=1 .
\end{array}\right.
$$

Не теряя в общности, можно положить $\gamma^{\prime}=0, \mu\left(S^{d-1}\right)=1$. При таком выборе распределение, отвечающее характеристической функции (1.3), является строго устойчивым для всех $\alpha \neq 1$. При этом в (1.1) $b_{n}=n^{1 / \alpha}$. Что касается случая $\alpha=1$, то строгая устойчивость имеет место, если

$$
\mathbf{a}^{(0)}=\int_{S^{d-1}} e \mu(\mathrm{d} e)=\mathbf{0} .
$$

В дальнейшем мы рассматриваем только устойчивые законы, допускающие следующее представление для своих характеристических функций:

$$
-\ln \widehat{s}(t)=\left\{\begin{array}{c}
\int_{S^{d-1}}|\langle t, e\rangle|^{\alpha}\left(1-\imath \operatorname{tg}\left(\frac{\alpha \pi}{2}\right) \operatorname{sign}\langle t, e\rangle\right) \mu(\mathrm{d} e), \\
\text { если } \quad \alpha \in(0,1) \cup(1,2), \\
\int_{S^{d-1}}|\langle t, e\rangle|\left(1+\imath \frac{2}{\pi} \operatorname{sign}\langle t, e\rangle \ln |\langle t, e\rangle|\right) \mu(\mathrm{d} e), \\
\text { если } \quad \alpha=1,
\end{array}\right.
$$

где $\mu\left(S^{d-1}\right)=1$.

Из (1.3) и (1.4) следует, что имеет место формула обрашения

$$
s(x)=(2 \pi)^{-d} \int_{\mathbf{R}^{d}} \mathrm{e}^{-\imath\langle t, x\rangle} \widehat{s}(t) \mathrm{d} t .
$$


При этом, как легко заметить,

$$
\sup _{x} s(x)<\infty
$$

K сожалению, не существует явных формул для плотностей устойчивых распределений за исключением нескольких частных случаев, которые малоинтересны с точки зрения многомерного анализа. Более того, в отличие от одномерного случая очень мало известно даже об асимптотических свойствах устойчивых плотностей.

Первый результат, относящийся к асимптотическому анализу устойчивых законов, был получен Н. Б. Калинаускайте [2], которая рассмотрела случай сферически инвариантных устойчивых плотностей, называемых также субаауссовскими (см., например, [1, гл. 2]).

Серьезный прогресс был достигнут С. В. Архиповым [3], который рассмотрел случай, когда $\mu$ абсолютно непрерывна относительно поверхностной меры Лебега $\chi_{d-1}$ на единичной сфере $S^{d-1}$, а спектральная плотность $\frac{\mathrm{d} \mu}{\mathrm{d} \chi_{d-1}}$ удовлетворяет некоторым условиям гладкости. При этом «длина» получающегося асимптотического разложения зависит от степени гладкости спектральной плотности.

K сожалению, автор может сослаться только на эти две работы. Дело в том, что асимптотическая теория устойчивых распределений очень слабо развита. Многие совершенно естественные вопросы до сих пор остаются без ответа. В частности, ничего не известно об асимптотическом поведении устойчивых распределений, отвечающих скольконибудь общим дискретным спектральным мерам. Неясно также, как возможные «дыры» в носителе спектральной меры сказываются на асимптотических формулах для соответствующих плотностей.

Целью данной работы является выяснение роли, которую играет положение носителя спектральной меры. Пусть поначалу $d=1$. Тогда спектральная мера $\mu$ сосредоточена на $\{-1,1\}$. Предположим, что $\mu(\{-1\})=0, \mu(\{1\})=1$. Тогда

$$
\mathscr{S}_{1}=\operatorname{supp} s(x)=\left\{\begin{array}{lll}
\mathbf{R}_{+}^{1}, & \text { если } & \alpha \in(0,1), \\
\mathbf{R}^{1}, & \text { если } & \alpha \in[1,2)
\end{array}\right.
$$

(см., например, замечание 4 по поводу теоремы 2.2 .3 в книге [4]). Естественно называть такие устойчивые распределения односторонними. Хорошо известно, что односторонняя плотность $s(x)$ правильно изменяется с показателем $-(\alpha+1)$ при $x \rightarrow \infty$. Если же $x \rightarrow x_{-}=$ $\inf \left\{x: x \in \mathscr{S}_{1}\right\}$, то $s(x)$ очень быстро убывает. Асимптотические формулы и ряды для односторонней плотности $s(x)$ приведены в [5] (см. там теоремы 2.4.4, 2.4.6 и 2.4.7). Эти утверждения установлены с помощью контурного интегрирования аналитических функций. Недавно автор в 
работе [6] предложил другой метод, основанный на технике Крамера доказательства теорем о больших уклонениях. Удивительно, что классические предельные теоремы для сумм независимых одинаково распределенных случайных величин позволяют устанавливать чисто аналитические факты.

Возникают вопросы: (а) какие многомерные устойчивые распределения следует считать односторонними? (b) может ли техника Крамера быть перенесена на многомерньй случай?

Главная цель данной работы - ответить на эти вопросы.

Пусть отныне $d>1$. Через $S_{0}$ обозначим носитель $\mu$. Предположим, что $\operatorname{dim} S_{0}=d$. Рассмотрим минимальный выпуклый конус $\mathscr{K}_{+}$, содержащий $S_{0}$. Положим $S_{+}=S^{d-1} \cap \mathscr{K}_{+}$и $S_{-}=\{e:\langle e, \varepsilon\rangle \leqslant 0$ для всех $\left.\varepsilon \in S_{+}\right\}$. По аналогии с одномерным случаем мы называем устойчивое распределение односторонним, если $\operatorname{dim} S_{-}=d$.

Из предложения 2.3.7 в [1] и замечаний 2.2.2 и 2.2.3 в [4] легко следует, что (ср. (1.5))

$$
\mathscr{S}_{d}=\operatorname{supp} s(x)=\left\{\begin{array}{lll}
\mathscr{K}_{+}, & \text {если } & \alpha \in(0,1), \\
\mathbf{R}^{d}, & \text { если } & \alpha \in[1,2) .
\end{array}\right.
$$

Как и в [5], приходится различать случаи $\alpha \in(0,1), \alpha \in(1,2)$ и $\alpha=1$. Если $\alpha \neq 1$, то полученная асимптотика для $s(x)$ предполагает, что $x \in X^{\prime}$, где $X^{\prime}$ является замкнутым конусом. При этом если $\alpha \in(0,1)$, то $X^{\prime} \subset \mathscr{K}_{+}$и $|x| \rightarrow 0$. Если $\alpha \in(1,2)$, то $X^{\prime} \subset-\mathscr{K}_{+}$и $|x| \rightarrow \infty$. Если же $\alpha=1$, то $X^{\prime}$ является неким цилиндрическим подмножеством $\mathbf{R}^{d}$, a $|x| \rightarrow \infty$.

Асимптотический анализ осуществляется с помощью метода, предложенного в [6]. Он оказывается очень эффективным при любой размерности пространства.

Следует подчеркнуть, что не предполагается никакой регулярности $\mu$. Так что, строго говоря, упомянутые выше результаты Архипова здесь неприменимы. Иными словамл, оказывается, что исследовать легкие «хвосты» распределений гораздо легче, чем типичные для устойчивых распределений тяжелые. Следует заметить, что впервые на этот факт обратила внимание Н. Б. Калинаускайте [7], которая пользовалась методом перевала. Однако язык классических предельных теорем лучше отвечает существу проблемы. В частности, классические предельные теоремы позволяют получать не только главные члены асимптотики, но и асимптотические ряды.

Работа построена следуюшим образом. Во втором и третьем разделах устанавливаются некоторые вспомогательные факты. Главный член асимптотики устойчивой плотности устанавливается в четвертом разделе. Асимптотические ряды приводятся в пятом разделе. 


\section{2. Вспомогательные предложения}

Условимся обозначать $e_{x}=|x|^{-1} x, x \in \mathbf{R}^{d}$. Далее, пусть $\mathscr{S}_{\alpha, \mu}$ означает устойчивое распределение с характеристической функцией (1.4). Рассмотрим два подобных распределения $\mathscr{S}_{\alpha, \mu_{i}}, i=1,2$. Наша цель оценить близость этих распределений в терминах близости между $\mu_{1}$ и $\mu_{2}$. При этом близость между распределениями понимается в смысле равномерной метрики $L_{\infty}$, тогда как близость между спектральными мерами измеряется с помощью метрики Прохорова. $\pi$. Случай симметричных ненормированных мер $\mu_{i}, i=1,2$, был рассмотрен в [8] (см. теорему 2.1 в этой работе). Для $i=1,2$ обозначим

$$
\Sigma_{i}=\Sigma_{i}(e)=\int_{S^{d-1}}|\langle e, \varepsilon\rangle|^{\alpha} \mu_{i}(\mathrm{~d} \varepsilon)
$$

и

$$
I_{i}=I_{i}(e)=\left\{\begin{array}{lll}
\int_{S^{d-1}}|\langle e, \varepsilon\rangle|^{\alpha} \operatorname{sign}\langle e, \varepsilon\rangle \mu_{i}(\mathrm{~d} \varepsilon), & \text { если } \quad \alpha \in(0,1) \cup(1,2), \\
\int_{S^{d-1}}\langle e, \varepsilon\rangle \ln |\langle e, \varepsilon\rangle| \mu_{i}(\mathrm{~d} \varepsilon), & \text { если } \quad \alpha=1 .
\end{array}\right.
$$

Ввиду (1.4) логарифмы характеристических функций могут быть переписаны в виде

$$
-\ln \widehat{s}_{i}(t)=|t|^{\alpha} \Sigma_{i}\left(e_{t}\right)-\imath|t|^{\alpha} c(\alpha) I_{i}\left(e_{t}\right), \quad i=1,2,
$$

где

$$
c(\alpha)=\left\{\begin{array}{lll}
\operatorname{tg} \frac{\alpha \pi}{2}, & \text { если } \quad \alpha \in(0,1) \cup(1,2), \\
-\frac{2}{\pi}, & \text { если } \quad \alpha=1 .
\end{array}\right.
$$

Положим

$$
M(e)=\Sigma_{1}(e) \vee \Sigma_{2}(e), \quad m(e)=\Sigma_{1}(e) \wedge \Sigma_{2}(e), \quad m=\min _{e \in S^{d-1}} m(e) .
$$

Если размерность носителей мер $\mu_{1}$ и $\mu_{2}$ равна $d$, то, очевидно, $m>0$.

Следуюшее предложение стоит сравнить с теоремой 2.1 в [8].

Лемма 2.1. Пусть устойчивье плотности $s_{i}(x)$ отвечают распределениям $\mathscr{S}_{\alpha, \mu_{i}}, i=1,2$. Если $m>0$, то

$$
\sup _{x}\left|s_{1}(x)-s_{2}(x)\right| \leqslant\left\{\begin{array}{lll}
K_{\alpha}\left(\pi\left(\mu_{1}, \mu_{2}\right)\right)^{\alpha \wedge 1}, & \text { eсли } \quad \alpha \in(0,1) \cup(1,2), \\
K_{1} \pi\left(\mu_{1}, \mu_{2}\right)\left|\ln \pi\left(\mu_{1}, \mu_{2}\right)\right|, & \text { если } \quad \alpha=1,
\end{array}\right.
$$

где $\pi$ - метрика Прохорова, а

$$
K_{\alpha}=\frac{1}{\alpha}\left(1+\left|\operatorname{tg} \frac{\alpha \pi}{2}\right|\right)(\alpha \vee 1) m^{-d / \alpha-1} 2^{2-d} \pi^{-d / 2} \frac{\Gamma(d / \alpha+1)}{\Gamma(d / 2)}, \quad \alpha \neq 1
$$


тогда как

$$
K_{1}=\frac{1}{\alpha}\left(1+\frac{\pi}{2}\right) m^{-d / \alpha-1} 2^{2-d} \pi^{-d / 2} \frac{\Gamma(d / \alpha+1)}{\Gamma(d / 2)} \quad c \quad \alpha=1 .
$$

Д о к а з а т е л ь с т в о. Из (2.1) и формулы обрашения следует, что

$$
\delta=\sup _{x}\left|s_{1}(x)-s_{2}(x)\right| \leqslant(2 \pi)^{-d} \int_{\mathbf{R}^{d}}\left|\mathrm{e}^{-|t|^{\alpha}\left(\Sigma_{1}-\imath c(\alpha) I_{1}\right)}-\mathrm{e}^{-|t|^{\alpha}\left(\Sigma_{2}-\imath c(\alpha) I_{2}\right)}\right| \mathrm{d} t .
$$

Замена переменных $t=r e, r>0, e \in S^{d-1}$, приводит к соотношению

$$
\delta \leqslant(2 \pi)^{-d} \int_{S^{d-1}} \chi_{d-1}(\mathrm{~d} e)\left(\int_{0}^{\infty} r^{d-1}\left|\mathrm{e}^{-r^{\alpha}\left(\Sigma_{1}-\imath c(\alpha) I_{1}\right)}-\mathrm{e}^{-r^{\alpha}\left(\Sigma_{2}-\imath c(\alpha) I_{2}\right)}\right| \mathrm{d} r\right)
$$

где $\chi_{d-1}$ - поверхностная мера Лебега на $S^{d-1}$. Хорошо известно (см., например, $[9$, гл. IV,$\S 10])$, что

$$
c_{d}=\int_{S^{d-1}} \chi_{d-1}(\mathrm{~d} e)=\frac{2 \pi^{d / 2}}{\Gamma(d / 2)} .
$$

Положим

$$
J(e)=\int_{0}^{\infty} r^{d / \alpha-1}\left|\mathrm{e}^{-r\left(\Sigma_{1}-\imath c(\alpha) I_{1}\right)}-\mathrm{e}^{-r\left(\Sigma_{2}-\imath c(\alpha) I_{2}\right)}\right| \mathrm{d} r
$$

Очевидно,

$$
\delta \leqslant \alpha^{-1}(2 \pi)^{-d} \int_{S^{d-1}} J(e) \chi_{d-1}(\mathrm{~d} e) .
$$

Далее положим

$$
I(e)=\left\{\begin{array}{lll}
I_{1}(e)-I_{2}(e), & \text { если } & m(e)=\Sigma_{2}(e), \\
I_{2}(e)-I_{1}(e), & \text { если } & m(e)=\Sigma_{1}(e) .
\end{array}\right.
$$

Тогда

$$
J(e)=\int_{0}^{\infty} r^{d / \alpha-1} \mathrm{e}^{-r m(e)}|1-\exp (-r[M(e)-m(e)]+\operatorname{rrc}(\alpha) I(e))| \mathrm{d} r .
$$

Поскольку для $\alpha>0$

$$
1-\mathrm{e}^{-a+\imath b} \leqslant 1-\mathrm{e}^{-a}+\left|1-\mathrm{e}^{\imath b}\right| \leqslant a+|b|,
$$

TO

$$
J(e) \leqslant([M(e)-m(e)]+|c(\alpha)||I(e)|) m^{-d / \alpha-1} \Gamma\left(d \alpha^{-1}+1\right) .
$$

Очевидно, что

$$
M(e)-m(e)=\left|\Sigma_{1}-\Sigma_{2}\right|
$$


В силу (2.2)

$$
\begin{aligned}
\delta \leqslant K^{\prime} & \left(\int_{S^{d-1}}\left|\Sigma_{1}(e)-\Sigma_{2}(e)\right| \chi_{d-1}(\mathrm{~d} e)\right. \\
& \left.\quad+|c(\alpha)| \int_{S^{d-1}}\left|I_{1}(e)-I_{2}(e)\right| \chi_{d-1}(\mathrm{~d} e)\right)
\end{aligned}
$$

где

$$
K^{\prime}=\alpha^{-1} m^{-d / \alpha-1}(2 \pi)^{-d} \Gamma\left(d \alpha^{-1}+1\right) .
$$

Ясно, что функции $h_{1}(z)=|z|^{\alpha},-1 \leqslant z \leqslant 1$, и $h_{2}(z)=|z|^{\alpha} \operatorname{sign} z,-1 \leqslant$ $z \leqslant 1$, имеют одинаковый модуль непрерывности $\omega(t)=(\alpha \vee 1) t^{\alpha \wedge 1}$. Рассмотрим семейство функций, заданных на $S^{d-1}$ следующим образом:

$$
\begin{aligned}
& H_{1 e}(\varepsilon)=h_{1}(\langle e, \varepsilon\rangle)=|\langle e, \varepsilon\rangle|^{\alpha}, \\
& H_{2 e}(\varepsilon)=h_{2}(\langle e, \varepsilon\rangle)=|\langle e, \varepsilon\rangle|^{\alpha} \operatorname{sign}\langle e, \varepsilon\rangle, \quad e \in S^{d-1} .
\end{aligned}
$$

Легко видеть, что эти функции равностепенно непрерывны. Более точно,

$$
\sup _{\left|\varepsilon^{\prime}-\varepsilon^{\prime \prime}\right|<t}\left|H_{i e}\left(\varepsilon^{\prime}\right)-H_{i e}\left(\varepsilon^{\prime \prime}\right)\right| \leqslant(\alpha \vee 1) t^{\alpha \wedge 1}, \quad i=1,2 .
$$

По лемме 1 в [10]

$$
\begin{aligned}
\sup _{e \in S^{d-1}}\left|\Sigma_{1}(e)-\Sigma_{2}(e)\right| & \leqslant \pi\left(\mu_{1}, \mu_{2}\right)+(\alpha \vee 1)\left(\pi\left(\mu_{1}, \mu_{2}\right)\right)^{\alpha \wedge 1} \\
& \leqslant 2(\alpha \vee 1)\left(\pi\left(\mu_{1}, \mu_{2}\right)\right)^{\alpha \wedge 1} .
\end{aligned}
$$

Аналогично,

$\sup _{e \in S^{d-1}}\left|I_{1}(e)-I_{2}(e)\right| \leqslant \begin{cases}2(\alpha \vee 1)\left(\pi\left(\mu_{1}, \mu_{2}\right)\right)^{\alpha \wedge 1}, & \text { если } \alpha \in(0,1) \cup(1,2), \\ 2 \pi\left(\mu_{1}, \mu_{2}\right)\left|\ln \pi\left(\mu_{1}, \mu_{2}\right)\right|, & \text { если } \alpha=1 .\end{cases}$

Возврашаясь к (2.3), получаем

$$
\delta \leqslant \begin{cases}2 c_{d} K^{\prime}(\alpha \vee 1)\left(1+\left|\operatorname{tg} \frac{\alpha \pi}{2}\right|\right)\left(\pi\left(\mu_{1}, \mu_{2}\right)\right)^{\alpha \wedge 1}, & \\ 2 c_{d} K^{\prime}\left(1+\frac{\pi}{2}\right) \pi\left(\mu_{1}, \mu_{2}\right)\left|\ln \pi\left(\mu_{1}, \mu_{2}\right)\right|, & \text { если } \quad \alpha \in(0,1) \cup(1,2),\end{cases}
$$

Лемма доказана.

Заметим, что утверждения леммы 2.1 и упомянутой теоремы 2.1 в [8] несколько сильнее основного утверждения в [11].

Теперь мы в состоянии установить формулу для производящей функции одностороннего устойчивого закона. 
Лемма 2.2. Пусть случайный вектор $\eta$ имеет устойчивое распределение $\mathscr{S}_{\alpha, \mu}, \alpha \neq 1$. Если $\operatorname{dim} S_{-}=d$, то производящая функиия $\eta$ допускает следующее представление:

$$
f(\lambda)=\exp \left(-\frac{1}{\cos (\alpha \pi / 2)} \int_{S_{0}}\langle-\lambda, \varepsilon\rangle^{\alpha} \mu(\mathrm{d} \varepsilon)\right)
$$

при условии, что

$$
\lambda \in \Lambda=\left\{\lambda: e_{\lambda} \in S_{-}\right\} .
$$

Д о к а з а т ель с т в о. Пусть случайные величины $\xi, \xi_{1}, \xi_{2}, \ldots$ независимы и одинаково распределены. Предположим, что распределение $\xi$ устойчиво и имеет характеристическую функцию вида

$$
\mathbf{E}^{\imath t \xi}=\exp \left(-|t|^{\alpha}\left(1-\imath \operatorname{tg}\left(\frac{\alpha \pi}{2}\right) \operatorname{sign} t\right)\right), \quad \alpha \neq 1, \quad t \in \mathbf{R}^{1} .
$$

Хорошо известно (см., например, предложение 1.2 .12 в [1] или теорему 2.6.1 в [4]), что производящая функция $\xi$ имеет вид

$$
\mathbf{E}^{t \xi}=\exp \left(-\frac{(-t)^{\alpha}}{\cos (\alpha \pi / 2)}\right), \quad t \leqslant 0 .
$$

Стоит подчеркнуть, что значение $\cos (\alpha \pi / 2)$ положительно или отрицательно в зависимости от того, принадлежит $\alpha$ интервалу $(0,1)$ или $(1,2)$.

Введем семейство случайных векторов

$$
\eta^{(n)}=\sum_{j=1}^{n} \sigma_{n, j} \xi_{j} e^{(j)}
$$

где $\sigma_{n, j}-$ положительные постоянные, а $e^{(j)} \in S_{0}$. Пусть мера $\mu_{n}$ сосредоточена на $e^{(1)}, \ldots, e^{(n)}$ и $\mu_{n}\left(\left\{e^{(j)}\right\}\right)=\sigma_{n, j}^{\alpha}$. Выберем $e^{(j)}$ и $\sigma_{n, j}$ таким образом, чтобы $\mu_{n}\left(S^{d-1}\right) \equiv 1$ и чтобы меры $\mu_{n}$, слабо сходились к $\mu$. Тогда

$$
\begin{aligned}
f_{n}(\imath t) & =\mathbf{E} \exp \left(\imath\left\langle t, \eta^{(n)}\right\rangle\right) \\
& =\exp \left(-\int_{S^{d-1}}|\langle t, e\rangle|^{\alpha}\left(1-\imath \operatorname{tg}\left(\frac{\alpha \pi}{2}\right) \operatorname{sign}\langle t, e\rangle\right) \mu_{n}(\mathrm{~d} e)\right) \\
& \longrightarrow \exp \left(-\int_{S^{d-1}}|\langle t, e\rangle|^{\alpha}\left(1-\imath \operatorname{tg}\left(\frac{\alpha \pi}{2}\right) \operatorname{sign}\langle t, e\rangle\right) \mu(\mathrm{d} e)\right) .
\end{aligned}
$$

В силу (2.4)

$$
\mathbf{E} \exp \left(\sigma_{n, j} \xi_{j}\left\langle\lambda, e^{(j)}\right\rangle\right)=\exp \left(-\sigma_{n, j}^{\alpha}\left\langle-\lambda, e^{(j)}\right\rangle^{\alpha}\right), \quad \lambda \in \Lambda,
$$

и, следовательно,

$$
\begin{aligned}
f_{n}(\lambda) & =\mathbf{E} \exp \left(\left\langle\lambda, \eta^{(n)}\right\rangle\right) \\
& =\exp \left(-\frac{1}{\cos (\alpha \pi / 2)} \int_{S^{d-1}}\langle-\lambda, \varepsilon\rangle^{\alpha} \mu_{n}(\mathrm{~d} \varepsilon)\right) \longrightarrow f(\lambda) .
\end{aligned}
$$

Лемма доказана. 
Лемма 2.3. Пусть случайный вектор $\eta$ имеет устойчивое распределение $\mathscr{S}_{1, \mu}$. Если $\operatorname{dim} S_{-}=d$, то производящая функиия $\eta$ допускает следующее представление:

$$
f(\lambda)=\exp \left(\frac{2}{\pi} \int_{S_{0}}\langle-\lambda, \varepsilon\rangle \ln \langle-\lambda, \varepsilon\rangle \mu(\mathrm{d} \varepsilon)\right)
$$

при условии, что

$$
\lambda \in \Lambda=\left\{\lambda: e_{\lambda} \in S_{-}\right\} .
$$

Дока за т е ль с т в о. Пусть случайные величины $\xi, \xi_{1}, \xi_{2}, \ldots$ одинаково распределены и независимы. Предположим, что распределение $\xi$ устойчиво и имеет характеристическую функцию вида

$$
\mathbf{E e}^{\imath t \xi}=\exp \left(-|t|\left(1+\imath \frac{2}{\pi}(\operatorname{sign} t) \ln |t|\right)\right), \quad t \in \mathbf{R}^{1} .
$$

Тогда производящая функция $\xi$ имеет вид (см., например, предложение 1.2 .12 в [1] или теорему 2.6 .1 в [4])

$$
\mathrm{E} e^{t \xi}=\exp \left(-\frac{2}{\pi} t \ln (-t)\right), \quad t \leqslant 0 .
$$

Рассмотрим семейство случайных векторов

$$
\eta^{(n)}=\sum_{j=1}^{n} \sigma_{n, j} \xi_{j} e^{(j)}+\frac{2}{\pi} \sum_{j=1}^{n} \sigma_{n, j} \ln \sigma_{n, j} e^{(j)},
$$

где, как и прежде, $\sigma_{n, j}$ являются положительными постоянными, а векторы $e^{(j)} \in S_{0}$. Пусть $\mu_{n}$ сосредоточена на $e^{(1)}, \ldots, e^{(n)}$, причем $\mu_{n}\left(\left\{e^{(j)}\right\}\right)=\sigma_{n, j}$. Выберем $e^{(j)}$ и $\sigma_{n, j}$ таким образом, чтобы выполнялись равенства

$$
\mu_{n}\left(S^{d-1}\right) \equiv 1
$$

и меры $\mu_{n}$ слабо сходились к $\mu$. Тогда

$$
\begin{aligned}
f_{n}(\imath t) & =\mathbf{E} \exp \left(\imath\left\langle t, \eta^{(n)}\right\rangle\right) \\
& =\exp \left(-\int_{S^{d-1}}|\langle t, e\rangle|\left(1+\imath \frac{2}{\pi} \operatorname{sign}\langle t, e\rangle \ln |\langle t, e\rangle|\right) \mu_{n}(\mathrm{~d} \varepsilon)\right) \\
& \longrightarrow \exp \left(-\int_{S^{d-1}}|\langle t, e\rangle|\left(1+\imath \frac{2}{\pi} \operatorname{sign}\langle t, e\rangle \ln |\langle t, e\rangle|\right) \mu(\mathrm{d} \varepsilon)\right) .
\end{aligned}
$$

Ввиду (2.5)

$$
f_{n}(\lambda) \rightarrow f(\lambda)
$$

Лемма доказана.

Строго говоря, достаточно иметь сходимость по распределению $\eta^{(n)} \stackrel{d}{\longrightarrow} \eta$, что гораздо слабее того, что дает лемма 2.3 . 


\section{3. Сопряженные распределения}

3.1. Случай $\alpha \in(0,1) \cup(1,2)$. Рассмотрим семейство плотностей

$$
p_{\lambda}(x)=(f(\lambda))^{-1} \mathrm{e}^{\left\langle\lambda, x_{i}\right.} s(x), \quad \lambda \in \Lambda .
$$

Плотность $p_{\lambda}(x)$ называется сопряженной к исходной плотности $s(x)$.

По лемме 2.2

$$
l(\lambda)=\ln f(\lambda)=c_{0}(\alpha) \int_{S_{0}}\langle-\lambda, \varepsilon\rangle^{\alpha} \mu(\mathrm{d} \varepsilon),
$$

где $c_{0}(\alpha)=-(\cos (\alpha \pi / 2))^{-1}$. Легко видеть, что

$$
l(\lambda)=|\lambda|^{\alpha} l\left(e_{\lambda}\right) .
$$

Вектор математических ожиданий, отвечающий $p_{\lambda}(x)$, дается формулой

$$
\mathbf{a}(\lambda)=\int x p_{\lambda}(x) \mathrm{d} x=\operatorname{grad} \ln f(\lambda),
$$

а ковариационная матрица $B(\lambda)$ представляет собой гессиан функции $\ln f(\lambda)$. Ввиду (3.2)

$$
\mathbf{a}(\lambda)=-c_{1}(\alpha) \int_{S_{0}} \varepsilon\langle-\lambda, \varepsilon\rangle^{\alpha-1} \mu(\mathrm{d} \varepsilon),
$$

a

$$
B(\lambda)=\left\|b_{i, k}(\lambda)\right\|_{i, k=1}^{d},
$$

где

$$
b_{i, k}(\lambda)=c_{2}(\alpha) \int_{S_{0}} \varepsilon_{i} \varepsilon_{k}\langle-\lambda, \varepsilon\rangle^{\alpha-2} \mu(\mathrm{d} \varepsilon), \quad i, k=1, \ldots, d,
$$

II

$$
c_{1}(\alpha)=\alpha c_{0}(\alpha), \quad c_{2}(\alpha)=\alpha(\alpha-1) c_{0}(\alpha)>0 .
$$

Очевидно, $B(\lambda)$ положительно определена для всех $\lambda \in \Lambda$. Это означает, что отображение

$$
\mathbf{a}(\lambda): \Lambda \rightarrow X
$$

взаимно однозначно.

Заметим, что

$$
\mathbf{a}(\lambda)=|\lambda|^{\alpha-1} \mathbf{a}\left(e_{\lambda}\right), \quad b_{i, k}(\lambda)=|\lambda|^{\alpha-2} b_{i, k}\left(e_{\lambda}\right), \quad i, k=1, \ldots, d .
$$

Это означает, что $X$ является конусом, порожденным множеством

$$
S_{x}=\left\{e: e=a\left(e_{\lambda}\right), e_{\lambda} \in S_{-}\right\} .
$$


Поскольку а $(e)$ есть результат усреднения $\varepsilon$ по множесгву $S_{0}$, то

$$
X \subset\left\{\begin{array}{lll}
\mathscr{K}_{+}, & \text {если } & \alpha \in(0,1), \\
-\mathscr{K}_{+}, & \text {если } & \alpha \in(1,2) .
\end{array}\right.
$$

Стоит заметить, что для любого замкнутого $S^{\prime} \subset S_{-}$

$$
\min _{e \in S^{\prime}} \operatorname{det} B(e)>0 \text {. }
$$

Пусть $F \subset \Lambda$ ограничено и замкнуто. Из (3.1) легко следует, что

$$
\int p_{\lambda}^{2}(x) \mathrm{d} x<\frac{f(2 \lambda)}{f(\lambda)} \sup _{x} s(x)<\infty
$$

откуда

$$
\sup _{\lambda \in F} \int p_{\lambda}^{2}(x) \mathrm{d} x<\infty .
$$

Тогда по тождеству Парсеваля

$$
\sup _{\lambda \in F} \int\left|\frac{f(\lambda+\imath t)}{f(\lambda)}\right|^{2} \mathrm{~d} t<\infty .
$$

Отсюда следует, в частности, что для $p_{n, \lambda}(x)$ равномерно по $\lambda \in F$ выполняются достаточные условия теорем 19.1 и 19.2 , доказанных в [12]. Таким образом, мы получаем следуюшую равномерную версию упомянутой теоремы 19.1 (ср. с доказательством теоремы 1 в [13])

$$
\sup _{\lambda \in F} \sup _{x}\left|p_{n, \lambda}(x)-n^{-d / 2} \phi_{\mathbf{0}, B(\lambda)}\left(n^{-1 / 2}[x-n \mathbf{a}(\lambda)]\right)\right|=o\left(n^{-d / 2}\right),
$$

где $\phi_{0, B(\lambda)}(u)$ - плотность нормального закона, имеющего нулевой вектор математических ожиданий и ковариационную матрицу $B(\lambda)$.

Рассмотрим уравнение

$$
x=\mathbf{a}(\lambda), \quad x \in X
$$

Очевидно, корень этого уравнения определяет отображение

$$
\mathbf{h}(x): X \rightarrow \Lambda
$$

которое является обратным к (3.3). Из (3.4) следует, что луч $X_{e}=$ $\{x: x=t \mathbf{a}(e), t>0\} \subset X$ отображается в луч $\Lambda_{e}=\{\lambda: \lambda=s e, s>0\}$, $e \in S_{-}$. Причем

$$
\mathbf{h}(t \mathbf{a}(e))=t^{1 /(\alpha-1)} e .
$$

Рассмотрим функцию уклонений, называемую также преобразованием Лежандра-Фенхеля функции $\ln f(\lambda)$ (см., например, $[14$, с. 26, 134]):

$$
L(x)=\sup _{\lambda \in \Lambda}(\langle\lambda, x\rangle-\ln f(\lambda))=\sup _{\lambda \in \Lambda}(\langle\lambda, x\rangle-l(\lambda)) .
$$


Легко видеть, что для $x \in X$

$$
L(x)=\langle\mathbf{h}(x), x\rangle-l(\mathbf{h}(x)) .
$$

Использование (3.2), (3.4) и (3.6) дает нам

$$
L(t \mathbf{a}(e))=(\alpha-1) l(e) t^{\alpha /(\alpha-1)} .
$$

Стоит заметить. что для всех $\alpha \in(0,1) \cup(1,2)$

$$
(\alpha-1) l(e)=(\alpha-1) c_{0}(\alpha) \int_{S_{0}}\langle-e, \varepsilon\rangle^{\alpha} \mu(\mathrm{d} \varepsilon)>0 .
$$

Заметим также, что в силу (3.4)

$$
\operatorname{det} B(t e)=t^{d(\alpha-2)} \operatorname{det} B(e), \quad e \in S_{-} .
$$

3.2. Случай $\alpha=1$. Рассмотрим семейство (3.1). На этот раз по лемме 2.2 (ср. (3.2))

$$
l(x)=\ln f(\lambda)=\frac{2}{\pi} \int_{S_{0}}\langle-\lambda, \varepsilon\rangle \ln \langle-\lambda, \varepsilon\rangle \mu(\mathrm{d} \varepsilon) .
$$

Легко видеть, что вектор математических ожиданий и ковариационная матрица, отвечающие $p_{\lambda}(x)$, задаются формулами

$$
\mathbf{a}(\lambda)=-\frac{2}{\pi}\left(\int_{S_{0}} \varepsilon \ln \langle-\lambda, \varepsilon\rangle \mu(\mathrm{d} \varepsilon)+\int_{S_{0}} \varepsilon \mu(\mathrm{d} \varepsilon)\right)
$$

и

$$
B(\lambda)=\left\|b_{i, k}(\lambda)\right\|_{i, k=1}^{d}
$$

где

$$
b_{i, k}(\lambda)=\frac{2}{\pi} \int_{S_{0}} \varepsilon_{i} \varepsilon_{k}\langle-\lambda, \varepsilon\rangle^{-1} \mu(\mathrm{d} \varepsilon), \quad i, k=1, \ldots, d .
$$

Как и прежде, $B(\lambda)$ положительно определена для любого $\lambda \in \operatorname{int} \Lambda$. Так что отображение (3.3) взаимно однозначно.

Заметим, что

$$
\mathbf{a}(\lambda)=\mathbf{a}\left(e_{\lambda}\right)-\frac{2}{\pi}(\ln |\lambda|) \mathbf{a}^{(0)},
$$

где

$$
\mathbf{a}^{(0)}=\int_{S_{0}} \varepsilon \mu(\mathrm{d} \varepsilon) \neq \mathbf{0} .
$$

При этом (ср. (3.8))

$$
B(\lambda)=|\lambda|^{-1} B\left(e_{\lambda}\right), \quad \operatorname{det} B(\lambda)=|\lambda|^{-d} \operatorname{det} B\left(e_{\lambda}\right) .
$$


Рассмотрим отображение (3.3). Как и прежде, корень уравнения

$$
x=\mathbf{a}(\lambda), \quad x \in X
$$

определяет отображение

$$
\mathbf{h}(x): X \rightarrow \Lambda
$$

являющееся обратным к (3.3). Из (3.10) следует, что $\mathbf{h}(x)$ отображает прямую $X_{\varepsilon}=\left\{x: x=\mathbf{a}(\varepsilon)-t \mathbf{a}^{(0)}, t \in \mathbf{R}^{1}\right\} \subset X$ в луч $\Lambda_{\varepsilon}=\{\lambda: \lambda=s \varepsilon$, $s>0\}, \varepsilon \in S_{-}$, где $\lambda=\mathrm{e}^{\pi t / 2} \varepsilon$. Обозначим через $A_{0}$ проекцию $\{x: x=$ $\left.\mathbf{a}(\varepsilon), \varepsilon \in S_{-}\right\}$на подпространство $\mathscr{L}_{d-1}=\left\{x \in \mathbf{R}^{d}:\left\langle x, \mathbf{a}^{(0)}\right\rangle=0\right\}$. Тогда $X$ можно представить в виде

$$
X=A_{0} \times\left\{x: x=-t \mathbf{a}^{(0)}, t \in \mathbf{R}^{1}\right\} .
$$

Здесь функция уклонений $L(x)$ при $x \in X$ имеет вид

$$
L(x)=L\left(\mathbf{a}(\varepsilon)-t \mathbf{a}^{(0)}\right)=-\left\langle-\mathbf{a}^{(0)}, \varepsilon\right\rangle \mathrm{e}^{\pi t / 2}, \quad \varepsilon \in S_{-} .
$$

\section{4. Большие уклонения}

4.1. Случай $\alpha \in(0,1) \cup(1,2)$. Пусть $\eta^{(j)}, j=1, \ldots, n$, являются независимыми копиями случайного вектора $\eta$, имеющего одностороннее устойчивое распределение с $\alpha \neq 1$. Благодаря строгой устойчивости имеем

$$
\eta \stackrel{d}{=} n^{-1 / \alpha}\left(\eta^{(1)}+\cdots+\eta^{(n)}\right)
$$

или, в терминах плотностей,

$$
s(x)=n^{d / \alpha} s_{n}\left(n^{1 / \alpha} x\right),
$$

где $s_{n}(x)$ является $n$-й сверткой $s(x)$.

Именно соотношение (4.1) позволяет нам применить мощную технику сопряженных распределений.

Если $\alpha \in(0,1), x \in \mathscr{K}_{+},|x| \rightarrow 0, n \rightarrow \infty$, то значения $n^{1 / \alpha} x$ лежат в области больших уклонений для $n^{-1 / \alpha}\left(\eta^{(1)}+\cdots+\eta^{(n)}\right)$. Если же $\alpha \in(1,2)$, то большие уклонения возникают, когда $-x \in \mathscr{K}_{+}, x \rightarrow \infty, n \rightarrow \infty$.

Рассмотрим преобразование Крамера

$$
s_{n}(x)=f^{n}(\lambda) \mathrm{e}^{-\langle\lambda, x\rangle} p_{n, \lambda}(x), \quad \lambda \in \Lambda,
$$

где $p_{n, \lambda}(x)$ означает $n$-ю свертку $p_{\lambda}(x)$.

Из (3.5) следует, что

$$
\sup _{\lambda \in F}\left|p_{n, \lambda}(n \mathbf{a}(\lambda))-(2 \pi n)^{-d / 2}(\operatorname{det} B(\lambda))^{-1 / 2}\right|=o\left(n^{-d / 2}\right) .
$$


Если $x=t \mathbf{a}(e), n^{1 / \alpha} x=n \mathbf{a}(\lambda), e \in S_{-}$, то в силу $(3.6)$

$$
\lambda=\mathbf{h}\left(n^{1 / \alpha-1} x\right)=t^{1 /(\alpha-1)} n^{-1 / \alpha} e .
$$

Следовательно, имея в виду (3.7) и (3.8), мы получаем

$$
\langle\lambda, \mathbf{a}(\lambda)\rangle-l(\lambda)=L\left(n^{1 / \alpha-1} x\right)=(\alpha-1) l(e) t^{\alpha /(\alpha-1)} n^{-1}
$$

и

$$
\operatorname{det} B\left(\mathbf{h}\left(n^{1 / \alpha-1} x\right)\right)=t^{d(\alpha-2) /(\alpha-1)} n^{-d(\alpha-2) / \alpha} \operatorname{det} B(e) .
$$

Из (4.2), (4.3) и (3.8) следует, что при $n \rightarrow \infty,|\lambda| \asymp 1$ (ср. с доказательством теоремы 1 в [13])

$$
\begin{aligned}
s(\operatorname{a}(e))= & n^{d / \alpha-d / 2}(2 \pi)^{-d / 2}(\operatorname{det} B(\lambda))^{-1 / 2} e^{-n(\langle\lambda, \mathbf{a}(\lambda)\rangle-l(\lambda))}(1+o(1)) \\
= & (2 \pi)^{-d / 2}(\operatorname{det} B(e))^{-1 / 2} t^{-d(\alpha-2) /(2(\alpha-1))} \\
& \times \exp \left(-(\alpha-1) l(e) t^{\alpha /(\alpha-1)}\right)(1+o(1)) .
\end{aligned}
$$

Остается обосновать использование (3.5). Пусть $S^{\prime}-$ замкнутое подмножество int $S_{-}$. Очевидно, что

$$
X^{\prime}=\left\{x: x=t \mathbf{a}(e), t>0, e \in S^{\prime}\right\}
$$

является замкнутым подмножеством $X$. Положим $z=n^{1 / \alpha-1}$. Если $z \in X^{\prime}$, то существуют константы $c_{ \pm}>0$ такие, что

$$
c_{-}|x|^{-\alpha /(1-\alpha)} \leqslant n \leqslant c_{+}|x|^{-\alpha /(1-\alpha)} .
$$

Вспомним, что $|x| \rightarrow 0$, если $\alpha \in(0,1)$, тогда как $|x| \rightarrow \infty$, если $\alpha \in(1,2)$. Это означает, что $n \rightarrow \infty$ и, следовательно, использование упомянутой локальной теоремы вполне корректно.

Таким образом, мы приходим к следуюшему утверждению.

Теорема 4.1. Пусть $\operatorname{dim} S_{0}=\operatorname{dim} S_{-}=d$, a $S^{\prime}$ является замкнутьмм подмножеством int $S_{-}$. Пусть, далее,

$$
t \rightarrow\left\{\begin{array}{lll}
0, & \text { если } & \alpha \in(0,1), \\
\infty, & \text { если } & \alpha \in(1,2) .
\end{array}\right.
$$

Tогда

$$
s(\operatorname{ta}(e))=g(e) t^{\gamma_{d}} \exp \left(-g_{1}(e) t^{\alpha /(\alpha-1)}\right)(1+o(1))
$$

равномерно по $е \in S^{\prime}$. Здесь

$$
\gamma_{d}=\frac{d(\alpha-2)}{2(\alpha-1)}
$$

mогда как

$$
g(e)=(2 \pi)^{-d / 2}(\operatorname{det} B(e))^{-1 / 2},
$$

$a$

$$
g_{1}(e)=\frac{1-\alpha}{\cos (\alpha \pi / 2)} \int_{S_{0}}\langle-e, \varepsilon\rangle^{\alpha} \mu(\mathrm{d} \varepsilon)>0 .
$$


Стоит подчеркнуть, что установленная асимптотика действует в некотором замкнутом конусе. Если $\alpha \in(0,1)$, то этот конус содержится в $\mathscr{K}_{+}$. Если же $\alpha \in(1,2)$, то он содержится в $-\mathscr{K}_{+}$.

4.2. Случай $\alpha=1$. Пусть $\eta^{(i)}, j=1, \ldots, n$, являются независимыми копиями случайного вектора $\eta$, имеющего одностороннее устойчивое распределение с $\alpha=1$. Ввиду (1.4) имеем

$$
\eta \stackrel{d}{=} n^{-1}\left(\eta^{(1)}+\cdots+\eta^{(n)}\right)-\frac{2}{\pi}(\ln n) \mathbf{a}^{(0)}
$$

или, в терминах плотностей,

$$
s(x)=n^{d} s_{n}\left(n\left[x+\frac{2}{\pi}(\ln n) \mathbf{a}^{(0)}\right]\right) .
$$

Пусть $x$ имеет вид

$$
x=\mathbf{a}(\varepsilon)-\frac{2}{\pi} t \mathbf{a}^{(0)}, \quad \varepsilon \in S_{-} .
$$

Рассмотрим уравнение

$$
\mathbf{a}(\lambda)=x+\frac{2}{\pi}(\ln n) \mathbf{a}^{(0)}, \quad x \in X .
$$

Из (3.10) получаем

$$
e_{\lambda}=\varepsilon, \quad|\lambda|=n^{-1} \mathrm{e}^{t} .
$$

Если $0<c_{-} \leqslant|\lambda| \leqslant c_{+}<\infty$ и $t \rightarrow \infty$, то $n \rightarrow \infty$. Так что можно применить локальную предельную теорему. $\mathrm{C}$ помощью (4.5), (4.2), $(4.3),(3.12)$ и $(3.11)$ получаем

$$
\begin{aligned}
s\left(\mathbf{a}(\varepsilon)-\frac{2}{\pi} t \mathbf{a}^{(0)}\right)= & n^{d} s_{n}(n \mathbf{a}(\lambda))=n^{d / 2}|\lambda|^{d / 2}(2 \pi)^{-d / 2}(\operatorname{det} B(\varepsilon))^{-1 / 2} \\
& \times \exp \left(-\mathrm{e}^{t}\left\langle-\mathbf{a}^{(0)}, \varepsilon\right\rangle\right)(1+o(1)),
\end{aligned}
$$

или

$$
\begin{aligned}
s\left(\mathbf{a}(\varepsilon)-\frac{2}{\pi} t \mathbf{a}^{(0)}\right)= & (2 \pi)^{-d / 2}(\operatorname{det} B(\varepsilon))^{-1 / 2} \\
& \times \exp \left(-\mathrm{e}^{t}\left\langle-\mathbf{a}^{(0)}, \varepsilon\right\rangle+\frac{d}{2} t\right)(1+o(1)) .
\end{aligned}
$$

Таким образом, мы приходим к следуюшему утверждению.

Теорема 4.2. Пусть $\operatorname{dim} S_{0}=\operatorname{dim} S_{-}=d$, a $S^{\prime}$ является замкнуmьмм подмножеством int $S_{-}$. Тогда при $t \rightarrow \infty$

$$
\begin{aligned}
s\left(\mathbf{a}(\varepsilon)-\frac{2}{\pi} \mathbf{t} \mathbf{a}^{(0)}\right) & (2 \pi)^{-d / 2}(\operatorname{det} B(\varepsilon))^{-1 / 2} \\
& \times \exp \left(-\mathrm{e}^{t}\left\langle-\mathbf{a}^{(0)}, \varepsilon\right\rangle+\frac{d}{2} t\right)(1+o(1))
\end{aligned}
$$

равномерно по $\varepsilon \in S^{\prime}$. 
Заметим, что

$$
x_{t}(\varepsilon)=\mathbf{a}(\varepsilon)-\frac{2}{\pi} t \mathbf{a}^{(0)}, \quad t \rightarrow \infty,
$$

означает, что

$$
e_{x_{t}(\varepsilon)} \rightarrow-e_{\mathbf{a}^{(0)}}
$$

равномерно по $\varepsilon \in S^{\prime}$. Так что в отличие от теоремы 4.1 множество допустимых $x$ в теореме 4.2 асимптотически очень узкое.

\section{5. Асимптотические разложения}

Для любого $d$-мерного вектора $\mathbf{n}=\left(n_{1}, \ldots, n_{d}\right)$, компонентами которого служат натуральные числа, обозначим

$$
\mathbf{n} !=n_{1} ! \cdots n_{d} !, \quad|\mathbf{n}|=n_{1}+\cdots+n_{d} .
$$

Далее, для любого $z=\left(z_{1}, \ldots, z_{d}\right)$ положим

$$
z^{\mathbf{n}}=z_{1}^{n_{1}} \cdots z_{d}^{n_{d}} .
$$

Рассмотрим однородные полиномы степени $|\mathbf{n}|$, определяемые следующим образом:

$$
\Gamma_{m}(z ; \lambda)=m ! \sum_{|\mathbf{n}|=m} \frac{\gamma_{\mathbf{n}}(\lambda)}{\mathbf{n} !} z^{\mathbf{n}}
$$

где

$$
\gamma_{\mathbf{n}}(\lambda)=\frac{\partial^{|\mathbf{n}|}}{\partial \lambda_{1}^{n_{1}} \cdots \partial \lambda_{d}^{n_{d}}} \ln f(\lambda)
$$

суть кумулянты сопряженной плотности $p_{\lambda}(x)$.

Пусть

$$
\Sigma_{j}(z)=\sum_{l=1}^{j} \frac{1}{l !} \sum_{\left(m_{1}, \ldots, m_{l}\right) \in \mathscr{M}_{j}} \frac{\Gamma_{m_{1}+2}(z)}{\left(m_{1}+2\right) !} \cdots \frac{\Gamma_{m_{l}+2}(z)}{\left(m_{l}+2\right) !}=\sum_{l=1}^{j} \frac{1}{l !} \Sigma_{l, j}(z),
$$

где

$$
\mathscr{M}_{j}=\left\{\left(m_{1}, \ldots, m_{l}\right): m_{r} \geqslant 1, r=1, \ldots, l, m_{1}+\cdots+m_{l}=j\right\} .
$$

Очевидно, $\Sigma_{j}(z)$ есть полином степени $3 j$, а $\Sigma_{l, j}(z)$ есть однородный полином степени $j+2 l$. В частности, (см., например, формулу (7.6) в [12])

$$
\left\{\begin{array}{l}
\Sigma_{1}(z)=\frac{1}{3 !} \Gamma_{3}(z), \\
\Sigma_{2}(z)=\frac{1}{4 !} \Gamma_{4}(z)+\frac{1}{2} \Gamma_{3}^{2}(z), \\
\Sigma_{3}(z)=\frac{1}{5 !} \Gamma_{5}(z)+\frac{1}{3 !} \frac{1}{4 !} \Gamma_{3}(z) \Gamma_{4}(z)+\left(\frac{1}{3 !}\right)^{4} \Gamma_{3}^{3}(z) .
\end{array}\right.
$$


Для любого полинома

$$
P_{r}(z)=\sum_{|\mathbf{i}| \leqslant r} p_{\mathbf{i}} z^{\mathbf{i}}, \quad \mathbf{i}=\left(i_{1}, \ldots, i_{d}\right),
$$

определим дифференциальный оператор

$$
P(D)=\sum_{|\mathbf{i}| \leqslant r} p_{\mathbf{i}} \frac{\partial^{|\mathbf{i}|}}{\partial \lambda_{1}^{i_{1}} \cdots \partial \lambda_{d}^{i_{d}}}
$$

По теореме 19.2 в [12] для любого $m \geqslant 1$ (ср. с доказательством теоремы 1 B [13])

$$
\begin{gathered}
\sup _{\lambda \in F} \sup _{u} \mid n^{-d / 2} p_{n, \lambda}\left(n \mathbf{a}(\lambda)+n^{1 / 2} u\right)-\phi_{\mathbf{0}, B(\lambda)}(u)-n^{-1 / 2} \Sigma_{1}(-D) \phi_{\mathbf{0}, B(\lambda)}(u) \\
-\cdots-n^{-m / 2} \Sigma_{m}(-D) \phi_{\mathbf{0}, B(\lambda)}(u) \mid=o\left(n^{-m / 2}\right) .
\end{gathered}
$$

При этом

$$
q_{j}(u ; \lambda)=\left(\phi_{\mathbf{0}, B(\lambda)}(u)\right)^{-1} \Sigma_{j}(-D) \phi_{\mathbf{0}, B(\lambda)}(u)
$$

является полиномом степени $j+2 l$. Если $j$ нечетно, то $q_{j, \lambda}(\mathbf{0})=0$.

Лемма 5.1. В принятых нами обозначениях $q_{2 j, \lambda}(\mathbf{0})$ является однородньм полиномом степени $j+2$ от $a_{11}(\lambda), a_{12}(\lambda), \ldots, a_{d d}(\lambda)$, где $a_{i k}(\lambda)$ суть элементь матриць $A(\lambda)=B^{-1}(\lambda)$.

Д ок а з а т е л с т в о. Положим для краткости

$$
R=-\frac{1}{2} x^{T} A(\lambda) x
$$

Обозначим

$$
g(x)=\mathrm{e}^{R}, \quad g_{i_{1} \ldots i_{n}}^{(n)}(x)=\frac{\partial^{n}}{\partial x_{i_{1}} \cdots \partial x_{i_{n}}} g(x), \quad 1 \leqslant i_{k} \leqslant d, \quad k=1, \ldots, n .
$$

Легко видеть, что

$$
\begin{aligned}
& g_{i}^{(1)}(x)=\mathrm{e}^{R}\left\langle a^{(i)}, x\right\rangle, \\
& g_{i k}^{(2)}(x)=\mathrm{e}^{R}\left(a_{i k}+\left\langle a^{(i)}, x\right\rangle\left\langle a^{(k)}, x\right\rangle\right), \\
& g_{i k j}^{(3)}(x)=\mathrm{e}^{R}\left(a_{i k}\left\langle a^{(j)}, x\right\rangle+a_{i j}\left\langle a^{(k)}, x\right\rangle+a_{k j}\left\langle a^{(i)}, x\right\rangle\right. \\
&\left.\quad+\left\langle a^{(i)}, x\right\rangle\left\langle a^{(k)}, x\right\rangle\left\langle a^{(j)}, x\right\rangle\right)
\end{aligned}
$$

и

$$
\begin{aligned}
g_{i k j l}^{(4)}(x)=\mathrm{e}^{R}( & a_{i k} a_{j k}+a_{i j} a_{k l}+a_{i l} a_{k j}+a_{i k}\left\langle a^{(j)}, x\right\rangle\left\langle a^{(l)}, x\right\rangle \\
& +a_{i j}\left\langle a^{(k)}, x\right\rangle\left\langle a^{(l)}, x\right\rangle+a_{i l}\left\langle a^{(k)}, x\right\rangle\left\langle a^{(j)}, x\right\rangle \\
& +a_{k j}\left\langle a^{(i)}, x\right\rangle\left\langle a^{(l)}, x\right\rangle+a_{k l}\left\langle a^{(i)}, x\right\rangle\left\langle a^{(j)}, x\right\rangle \\
& \left.+a_{j l}\left\langle a^{(i)}, x\right\rangle\left\langle a^{(k)}, x\right\rangle+\left\langle a^{(i)}, x\right\rangle\left\langle a^{(k)}, x\right\rangle\left\langle a^{(j)}, x\right\rangle\left\langle a^{(l)}, x\right\rangle\right),
\end{aligned}
$$


где $a^{(i)}=a^{(i)}(\lambda)$ обозначает $i$-ю строку матрицы $A(\lambda)$. Перепишем $g_{i k j}^{(3)}(x)$ и $g_{i k j l}^{(4)}(x)$ в виде

$$
\begin{aligned}
& g_{i k j}^{(3)}(x)=\mathrm{e}^{R}\left(Q_{i k j}^{(1)}(x)+Q_{i k j}^{(3)}(x)\right), \\
& g_{i k j l}^{(4)}(x)=\mathrm{e}^{R}\left(Q_{i k j l}^{(0)}(x)+Q_{i k j l}^{(2)}(x)+Q_{i k j l}^{(4)}(x)\right) .
\end{aligned}
$$

Здесь $Q_{\ldots}^{(i)}(x), i=0,1,2,3,4$, суть однородные полиномы степени $i$. В частности,

$$
Q_{i k j l}^{(0)}(x)=Q_{i k j l}^{(0)}(\mathbf{0})=a_{i k} a_{j k}+a_{i j} a_{k l}+a_{i l} a_{k j} .
$$

Сформулируем следующее предположение индукции:

$$
g_{i_{1} \ldots i_{2 m+1}}^{(2 m+1)}(x)=\mathrm{e}^{R} \sum_{r=0}^{m} Q_{i_{1} \ldots i_{2 m+1}}^{(2 r+1)}(x), \quad g_{i_{1} \ldots i_{2 m}}^{(2 m)}(x)=\mathrm{e}^{R} \sum_{r=0}^{m} Q_{i_{1} \ldots i_{2 m}}^{(2 r)}(x),
$$

где $Q_{i_{1} \ldots i_{2 m+1}}^{(2 r+1)}(x), r=0,1, \ldots, m$, и $Q_{i_{1} \ldots i_{2 m}}^{(2 r)}(x), r=1, \ldots, m$, суть однородные полиномы от $x$ степеней $2 r+1$ и $2 r$ соответственно, а $Q_{i_{1} \ldots i_{2 m}}^{(0)}(x)=$ $Q_{i_{1} \ldots i_{2 m}}^{(0)}(\mathbf{0})$ является однородным полиномом от $a_{11}, \ldots, a_{d d}$ степени $m$. Легко видеть, что

$$
Q_{i_{1} \ldots i_{2 m}}^{(2 r)}(x)\left\langle a^{\left(i_{2 m}\right)}, x\right\rangle Q_{i_{1} \ldots i_{2 m-1}}^{(2 r-1)}(x)+\frac{\partial}{\partial x_{i_{2 m}}} Q_{i_{1} \ldots i_{2 m-1}}^{(2 r+1)}(x)
$$

для $r=1, \ldots, m-1$, a

$$
Q_{i_{1} \ldots i_{2 m}}^{(2 m)}(x)=\left\langle a^{\left(i_{2 m}\right)}, x\right\rangle Q_{i_{1} \ldots i_{2 m-1}}^{(2 m-1)}(x), \quad Q_{i_{1} \ldots i_{2 m}}^{(0)}(x)=\frac{\partial}{\partial x_{i_{2 m}}} Q_{i_{1} \ldots i_{2 m-1}}^{(1)}(x) .
$$

Подобным же образом

$$
Q_{i_{1} \ldots i_{2 m+1}}^{(2 r+1)}(x)=\left\langle a^{\left(i_{2 m+1}\right)}, x\right\rangle Q_{i_{1} \ldots i_{2 m-1}}^{(2 r)}(x)+\frac{\partial}{\partial x_{i_{2 m+1}}} Q_{i_{1} \ldots i_{2 m}}^{(2 r+2)}(x)
$$

для $r=1, \ldots, m-1$, a

$$
Q_{i_{1} \ldots i_{2 m+1}}^{(2 m+1)}(x)=\left\langle a^{\left(i_{2 m+1}\right)}, x\right\rangle Q_{i_{1} \ldots i_{2 m-1}}^{(2 m-1)}(x)
$$

и

$$
Q_{i_{1} \ldots i_{2 m-1}}^{(1)}(x)=\frac{\partial}{\partial x_{i_{2 m+1}}} Q_{i_{1} \ldots i_{2 m}}^{(2)}(x)+\left\langle a^{\left(i_{2 m+1}\right)}, x\right\rangle Q_{i_{1} \ldots i_{2 m}}^{(0)}(x) .
$$

Это означает, что

$$
Q_{i_{1} \ldots i_{2 m+2}}^{(2 m+2)}(\mathbf{0})=a_{i_{2 m+1} i_{2 m}} Q_{i_{1}, i_{2 m}}^{(2 m)}(\mathbf{0}) .
$$

Остается вспомнить про предположение индукции. Лемма доказана. 
Полагая в $(5.2) u=\mathbf{0}$, получаем

$$
\sup _{\lambda \in F}\left|p_{n, \lambda}(n \mathbf{a}(\lambda))-\frac{1+\sum_{j=1}^{m} n^{-j} q_{2 j, \lambda}(\mathbf{0})}{n^{d / 2}(2 \pi)^{d / 2}(\operatorname{det} B(\lambda))^{1 / 2}}\right|=o\left(n^{-(d+m) / 2}\right) .
$$

Рассмотрим полиномы, фигурирующие в (5.1). Легко видеть, что

$$
\begin{aligned}
\Sigma_{l, 2 j}(z)=\sum_{\left(m_{1}, \ldots, m_{l}\right) \in \mathscr{M}_{2 j}\left|\mathbf{n}^{(1)}\right|=m_{1}+2, \ldots,\left|\mathbf{n}^{(l)}\right|=m_{l}+2} & \frac{\gamma_{\mathbf{n}^{(1)}}(\lambda)}{\mathbf{n}^{(1) !}} \cdots \frac{\gamma_{\mathbf{n}^{(l)}}(\lambda)}{\mathbf{n}^{(l) !}} \\
& \times z_{1}^{n_{11}+\cdots+n_{l 1}} \cdots z_{d}^{n_{1 d}+\cdots+n_{l d}},
\end{aligned}
$$

где $n_{j 1}, \ldots, n_{j d}$ являются компонентами векторов $\mathbf{n}^{(j)}, j=1, \ldots, l$. Обозначим

$$
r_{1}=n_{11}+\cdots+n_{l 1}, \ldots, r_{d}=n_{1 d}+\cdots+n_{l d}
$$

и $($ см. $(3.2)$ и $(3.9))$

$$
h\left(\lambda ; \mathbf{n}^{(1)}, \ldots, \mathbf{n}^{(l)}\right)=\prod_{j=1}^{l} c_{\left|\mathbf{n}^{(j)}\right|} \int_{S_{0}} \varepsilon^{\mathbf{n}^{(j)}}\langle-\lambda, \varepsilon\rangle^{\alpha-\left|\mathbf{n}^{(j)}\right|} \mu(\mathrm{d} \varepsilon)
$$

где

$$
c_{|\mathbf{n}|}(\alpha)=\left\{\begin{aligned}
(-1)^{\mathbf{n}} c_{0}(\alpha) \alpha(\alpha-1) \cdots(\alpha-|\mathbf{n}|+1), \\
\text { если } \quad \alpha \in(0,1) \cup(0,2), \\
\frac{\pi}{2}(n-2) !, \quad \text { если } \quad \alpha=1 .
\end{aligned}\right.
$$

Ввиду (5.1)

$$
\begin{aligned}
\Sigma_{l, 2 j}(z)= & |\lambda|^{l a-2(j+l)} \\
& \times \sum_{\left(m_{1}, \ldots, m_{l}\right) \in \mathscr{M}_{2 j}\left|\mathbf{n}^{(1)}\right|=m_{1}+2, \ldots,\left|\mathbf{n}^{(l)}\right|=m_{l}+2} h\left(e_{\lambda} ; \mathbf{n}^{(1)}, \ldots, \mathbf{n}^{(l)}\right) z_{1}^{r_{1}} \cdots z_{d}^{r_{d}} .
\end{aligned}
$$

Рассмотрим дифференциальный оператор $\Sigma_{l, 2 j}(-D)$. Он содержит производные порядка $r_{1}+\cdots+r_{d}=2(j+l)$. Следовательно,

$$
\begin{aligned}
|\lambda|^{2(j+l)-l \alpha} & \left.\frac{\Sigma_{l, 2 j}(-D) \phi_{\mathbf{0}, B(\lambda)}(u)}{\phi_{\mathbf{0}, B(\lambda)}(u)}\right|_{u=\mathbf{0}} h\left(e ; \mathbf{n}^{(1)}, \ldots, \mathbf{n}^{(l)}\right) \\
= & \left.\sum_{\left(m_{1}, \ldots, m_{l}\right) \in \mathscr{M}_{2 j}\left|\mathbf{n}^{(1)}\right|=m_{1}+2, \ldots,\left|\mathbf{n}^{(l)}\right|=m_{l}+2} h\left(\frac{1}{\phi_{\mathbf{0}, B(\lambda)}(u)} \frac{\partial^{2 j+2 l}}{\partial z_{1}^{r_{1}} \cdots \partial z_{d}^{r_{d}}} \phi_{\mathbf{0}, B(\lambda)}(u)\right)\right|_{u=\mathbf{0}} h\left(e ; \mathbf{n}^{(1)}, \ldots, \mathbf{n}^{(l)}\right) g_{r_{1} \ldots r_{d}}^{(2 j+2 l)}(\lambda) . \\
& \quad \sum_{\left(m_{1}, \ldots, m_{l}\right) \in \mathscr{M}_{2 j}\left|\mathbf{n}^{(1)}\right|=m_{1}+2, \ldots,\left|\mathbf{n}^{(l)}\right|=m_{l}+2}
\end{aligned}
$$


Заметим, что

$$
a_{i k}(\lambda)=|\lambda|^{2-\alpha} a_{i k}\left(e_{\lambda}\right), \quad i, k=1, \ldots, d .
$$

По определению частной производной функции $g$ (см. начало доказательства леммы 5.1)

$$
g_{r_{1} \ldots r_{d}}^{(2 j+2 l)}(\lambda)=|\lambda|^{(2-\alpha)(j+l)} g_{r_{1} \ldots r_{d}}^{(2 j+2 l)}\left(e_{\lambda}\right) .
$$

Следовательно,

$$
\left.\frac{\Sigma_{l, 2 j}(-D) \phi_{\mathbf{0}, B(\lambda)}(u)}{\phi_{\mathbf{0}, B(\lambda)}(u)}\right|_{u=\mathbf{0}}=|\lambda|^{-j \alpha} L_{l, j}^{(\alpha)}\left(e_{\lambda}\right)
$$

где

$$
L_{l, j}^{(\alpha)}(e)=\sum_{\left(m_{1}, \ldots, m_{l}\right) \in \mathscr{M}_{2 j}\left|\mathbf{n}^{(1)}\right|=m_{1}+2, \ldots,\left|\mathbf{n}^{(l)}\right|=m_{l}+2} h\left(e ; \mathbf{n}^{(1)}, \ldots, \mathbf{n}^{(l)}\right) g_{r_{1} \ldots r_{d}}^{(2 j+2 l)}(e) .
$$

Таким образом,

$$
\begin{aligned}
q_{2 j}(\mathbf{0} ; \lambda) & =\left.\frac{\Sigma_{2 j}(-D) \phi_{\mathbf{0}, B(\lambda)}(u)}{\phi_{\mathbf{0}, B(\lambda)}(u)}\right|_{u=\mathbf{0}} \\
& =|\lambda|^{-j \alpha} \sum_{l=1}^{j} \frac{1}{l !} L_{l, j}^{(\alpha)}\left(e_{\lambda}\right)=|\lambda|^{-j \alpha} L_{j}^{(\alpha)}\left(e_{\lambda}\right) .
\end{aligned}
$$

Принимая во внимание (5.3), (5.4), (3.8) и (3.11), получаем

$$
\sup _{e_{\lambda} \in S^{\prime}}\left|p_{n, \lambda}(n \mathbf{a}(\lambda))-\frac{1+\sum_{j=1}^{m} n^{-j}|\lambda|^{-j \alpha} L_{j}^{(\alpha)}(e)}{n^{d / 2}|\lambda|^{d(\alpha-2) / 2}(2 \pi)^{d / 2}\left(\operatorname{det} B\left(e_{\lambda}\right)\right)^{1 / 2}}\right|=o\left(n^{-(d+m) / 2}\right) .
$$

Пусть $\alpha \neq 1$. Если $x=t \mathbf{a}(\varepsilon), n^{1 / \alpha} x=n \mathbf{a}(\lambda), \varepsilon \in S_{--}$, то из (3.6) следует, что

$$
\lambda=\mathbf{h}\left(n^{1 / \alpha-1} x\right)=t^{1 /(\alpha-1)} n^{-1 / \alpha} \varepsilon .
$$

Если же $\alpha=1$ и $x=\mathbf{a}(\varepsilon)-(2 / \pi) t \mathbf{a}^{(0)}, \varepsilon \in S_{-}$, то $\lambda=n^{-1} \mathrm{e}^{t} \varepsilon$.

Принимая во внимание (4.4) и (4.6), мы приходим к следующему утвержденйю.

Теорема 5.1. Если $\alpha \in(0,1) \cup(1,2)$, то в условиях теоремь 4.1 для любого иелого $m \geqslant 1$ при $t \rightarrow \infty$

$$
\begin{aligned}
s(t \mathbf{a}(\varepsilon))= & g(\varepsilon) t^{\gamma_{d}} \exp \left(-g_{1}(\varepsilon) t^{\alpha /(\alpha-1)}\right) \\
& \times\left(1+\sum_{j=1}^{m} L_{j}^{(\alpha)}(\varepsilon) t^{-j \alpha /(\alpha-1)}+o\left(t^{-m \alpha /(\alpha-1)}\right)\right)
\end{aligned}
$$

равномерно по $\varepsilon \in S^{\prime}$. 
Если же $\alpha=1$, mо при $t \rightarrow \infty$

$$
\begin{aligned}
s\left(\mathbf{a}(\varepsilon)-\frac{2}{\pi} t \mathbf{a}^{(0)}\right)= & (2 \pi)^{-d / 2}(\operatorname{det} B(\varepsilon))^{-1 / 2} \exp \left(-\mathrm{e}^{t}\left\langle-\mathbf{a}^{(0)}, \varepsilon\right\rangle+\frac{d}{2} t\right) \\
& \times\left(1+\sum_{j=1}^{m} L_{j}^{(1)}(\varepsilon) \mathrm{e}^{-j t)}+o\left(\mathrm{e}^{-m t}\right)\right)
\end{aligned}
$$

равномерно по $\varepsilon \in S^{\prime}$.

Функиии $L_{j}^{(\alpha)}(\varepsilon), \varepsilon \in S^{d-1}$, били определеньл в (5.4).

Автор выражает глубокую благодарность коллективу математиков Университета Миннесоты, в котором была написана данная работа.

\section{СПИСОК ЛИТЕРАТУРЫ}

1. Samorodnitsky G., Taggu M.S. Stable Non-Gaussian Random Processes. New York: Chapman \& Hall, 1994, 632 p.

2. Калинаускайте $H$. Некоторые разложения многомерных симметрических устойчивых плотностей. - Литов. матем. сб., 1970, т. 10, № 4, с. 727-732.

3. Arkhipov $S$. $V$. The density function's asymptotic representation in the case of multidimensional strictly stable distributions. - Lecture Notes in Math., 1989, v. 1412, p. $1-21$.

4. Золотарев В. М. Одномерные устойчивые распределения. М.: Наука, 1983, 304 с.

5. Ибрагимов И.А., Линник Ю.В. Независимые и стационарно связанные величины. М.: Наука, 1965, 524 с.

6. Nagaev A. V. Asymptotic properties of stable densities and the asymmetric large deviation problems. - Statist. Probab. Lett., 2003, v. 61, № 4, p. 429-438.

7. Калинаускайте Н.Б. О показательном убывании некоторых многомерных плотностей. - Литов. матем. сб., 1971, т. 11, № 3, с. 557-562.

8. Davydov Yu., Nagaev A. V. On two approaches to approximation of multidimensional stable laws. - J. Multivariate Anal., 2002, v. 82, № 1, p. 210-239.

9. Швари Л. Анализ. Т. 1. М.: Мир, 1972, 824 с.

10. Davydov $Y u$. On the rate of strong convergence for convolutions. - J. Math. Sci., 1997, v. 83, № 3, p. 393-396.

11. Byczkowski T., Nolan J. P., Rajput B. Approximation of multidimensional stable densities. - J. Multivariate Anal., 1993, v. 46, № 1, p. 13-31.

12. Бхаттачария P. Н., Ранга Рао Р. Аппроксимация нормальным распределением и асимптотические разложения. М.: Наука, 1982, 286 с.

13. Боровков A. A., Рогозин Б. A. О центральной предельной теорем е в многомерном случае. - Теория вероятн. и ее примен., 1965, т. 10, в. 1, с. 61-69.

14. Dembo A., Zeitouni O. Large Deviations Techniques and Applications. Boston: Jones and Bartlett, 1993, $346 \mathrm{p}$. 